Trinity University

Digital Commons @ Trinity

Physics and Astronomy Faculty Research

Physics and Astronomy Department

$11-2017$

\title{
An Active Galactic Nucleus Caught in the Act of Turning Off and \\ On
}

Julia M. Comerford

R Scott Barrows

Francisco Müller Sánchez

Rebecca Nevin

Jenny E. Greene

See next page for additional authors

Follow this and additional works at: https://digitalcommons.trinity.edu/physics_faculty

Part of the Physics Commons

\section{Repository Citation}

Comerford, J.M., Barrows, R.S., Müller-Sánchez, F., Nevin, R., Greene, J.E., Pooley, D., Stern, D., \& Harrison, F.A. (2017). An active galactic nucleus caught in the act of turning off and on. The Astrophysical Journal, 849(2), 1-12. doi: 10.3847/1538-4357/aa8e4b

This Article is brought to you for free and open access by the Physics and Astronomy Department at Digital Commons @ Trinity. It has been accepted for inclusion in Physics and Astronomy Faculty Research by an authorized administrator of Digital Commons @ Trinity. For more information, please contact jcostanz@trinity.edu. 


\section{Authors}

Julia M. Comerford, R Scott Barrows, Francisco Müller Sánchez, Rebecca Nevin, Jenny E. Greene, David Pooley, Daniel Stern, and Fiona A. Harrison 


\title{
An Active Galactic Nucleus Caught in the Act of Turning Off and On
}

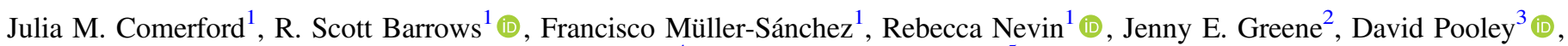 \\ Daniel Stern ${ }^{4}$ (D), and Fiona A. Harrison ${ }^{5}$ (D) \\ ${ }^{1}$ Department of Astrophysical and Planetary Sciences, University of Colorado, Boulder, CO 80309, USA \\ ${ }^{2}$ Department of Astrophysical Sciences, Princeton University, Princeton, NJ 08544, USA \\ ${ }^{3}$ Department of Physics and Astronomy, Trinity University, San Antonio, TX 78212, USA \\ ${ }^{4}$ Jet Propulsion Laboratory, California Institute of Technology, 4800 Oak Grove Drive, Pasadena, CA 91109, USA \\ ${ }^{5}$ California Institute of Technology, 1200 East California Boulevard, Pasadena, CA 91125, USA \\ Received 2017 July 10; revised 2017 August 28; accepted 2017 September 19; published 2017 November 6
}

\begin{abstract}
We present the discovery of an active galactic nucleus (AGN) that is turning off and then on again in the $z=0.06$ galaxy SDSS J1354+1327. This episodic nuclear activity is the result of discrete accretion events that could have been triggered by a past interaction with the companion galaxy that is currently located $12.5 \mathrm{kpc}$ away. We originally targeted SDSS J1354+1327 because its Sloan Digital Sky Survey spectrum has narrow AGN emission lines that exhibit a velocity offset of $69 \mathrm{~km} \mathrm{~s}^{-1}$ relative to systemic. To determine the nature of the galaxy and its velocity-offset emission lines, we observed SDSS J1354+1327 with Chandra/ACIS, Hubble Space Telescope/ Wide Field Camera 3, Apache Point Observatory optical longslit spectroscopy, and Keck/OSIRIS integral-field spectroscopy. We find a $\sim 10 \mathrm{kpc}$ cone of photoionized gas south of the galaxy center and a $\sim 1 \mathrm{kpc}$ semi-spherical front of shocked gas, which is responsible for the velocity offset in the emission lines, north of the galaxy center. We interpret these two outflows as the result of two separate AGN accretion events: the first AGN outburst created the southern outflow, and then $<10^{5}$ years later, the second AGN outburst launched the northern shock front. SDSS J1354+1327 is the galaxy with the strongest evidence for an AGN that has turned off and then on again, and it fits into the broader context of AGN flickering that includes observations of AGN light echoes.
\end{abstract}

Key words: galaxies: active - galaxies: nuclei

\section{Introduction}

When a supermassive black hole (SMBH) accretes a sufficient amount of gas, it shines as an active galactic nucleus (AGN). However, this is not a continuous process; SMBHs can turn on and off as AGNs depending on their accretion histories. A local example is the SMBH in the Milky Way, which is currently quiescent, but diffuse bubbles of gamma-ray emission extending $\sim 10 \mathrm{kpc}$ above and below the Galactic plane indicate that the SMBH was active $<10^{7}$ years ago (Su et al. 2010). Evidence of this past activity is also seen in X-ray reflection off galactic center molecular clouds (e.g., Koyama et al. 1996; Zhang et al. 2015).

Typical AGN variability timescales have been inferred to be $10^{4}-10^{5}$ years between turning on and turning off (e.g., Schawinski et al. 2015). This time variability is linked to accretion of clumpy interstellar medium (ISM) in individual events, as seen in simulations of SMBH accretion and the corresponding energetic AGN outbursts (e.g., Hopkins \& Quataert 2010; Gabor \& Bournaud 2013, 2014). Because observable emission signatures can linger for up to $10^{5}$ years after an AGN has turned off in a galaxy, this leads to observable light echoes from past AGN activity, which are seen in systems such as voorwerpen and changing-look AGNs (e.g., Tremonti et al. 2007; Lintott et al. 2009; Förster Schreiber et al. 2014; Keel et al. 2015; LaMassa et al. 2015; Runnoe et al. 2016; Schirmer et al. 2016).

Here, we present a galaxy that has evidence of two discrete AGN outburst events. SDSS J135429.05+132757.2 (hereafter SDSS J1354 +1327 , located at $z=0.06$ ) was originally noted for its velocity-offset narrow emission lines. By analogy to double-peaked narrow emission lines used to identify dual AGN candidates (e.g., Liu et al. 2010; Comerford et al. 2011;
Barrows et al. 2012, 2013; Fu et al. 2012; McGurk et al. 2015; Müller-Sánchez et al. 2015), single-peaked narrow AGN emission lines that exhibit a velocity offset relative to systemic can be used to select offset AGN candidates (Comerford et al. 2009, 2013). Followup observations of some of these galaxies that exhibit velocity-offset AGN emission lines in their Sloan Digital Sky Survey (SDSS) spectra have revealed that the velocity-offset emission lines can be produced by offset AGNs or by shocked gas in outflows or inflows (Allen et al. 2015; Barrows et al. 2016; Müller-Sánchez et al. 2016).

To determine the nature of SDSS J1354+1327, we observed the galaxy with the Chandra X-ray Observatory, Hubble Space Telescope (HST) imaging, Apache Point Observatory Dual Imaging Spectrograph (APO/DIS) optical longslit spectroscopy, and adaptive optics-assisted Keck OH-Suppressing InfraRed Imaging Spectrograph (Keck/OSIRIS) integral-field spectroscopy (IFS). We conclude that SDSS J1354+1327 is the best observational example of an AGN turning off and then on, with a relic outflow to the south and a new AGN outburst north of the galaxy center (Figure 1, left panel). We find that the velocity-offset emission lines are produced by shocks emanating from the new AGN outburst.

The paper is organized as follows. In Section 2, we present our observations, including space-based and ground-based observations of SDSS J1354+1327 and analyses of these data. In Section 3, we combine the observations to interpret the nature of the emission sources in SDSS J1354+1327 and the nature of its companion galaxy. In Section 4, we determine the nature of SDSS J1354+1327 itself. In Section 5, we present our conclusions. We assume a Hubble constant $H_{0}=$ $70 \mathrm{~km} \mathrm{~s}^{-1} \mathrm{Mpc}^{-1}, \Omega_{m}=0.3$, and $\Omega_{\Lambda}=0.7$ throughout, and all distances are given in physical (not comoving) units. 

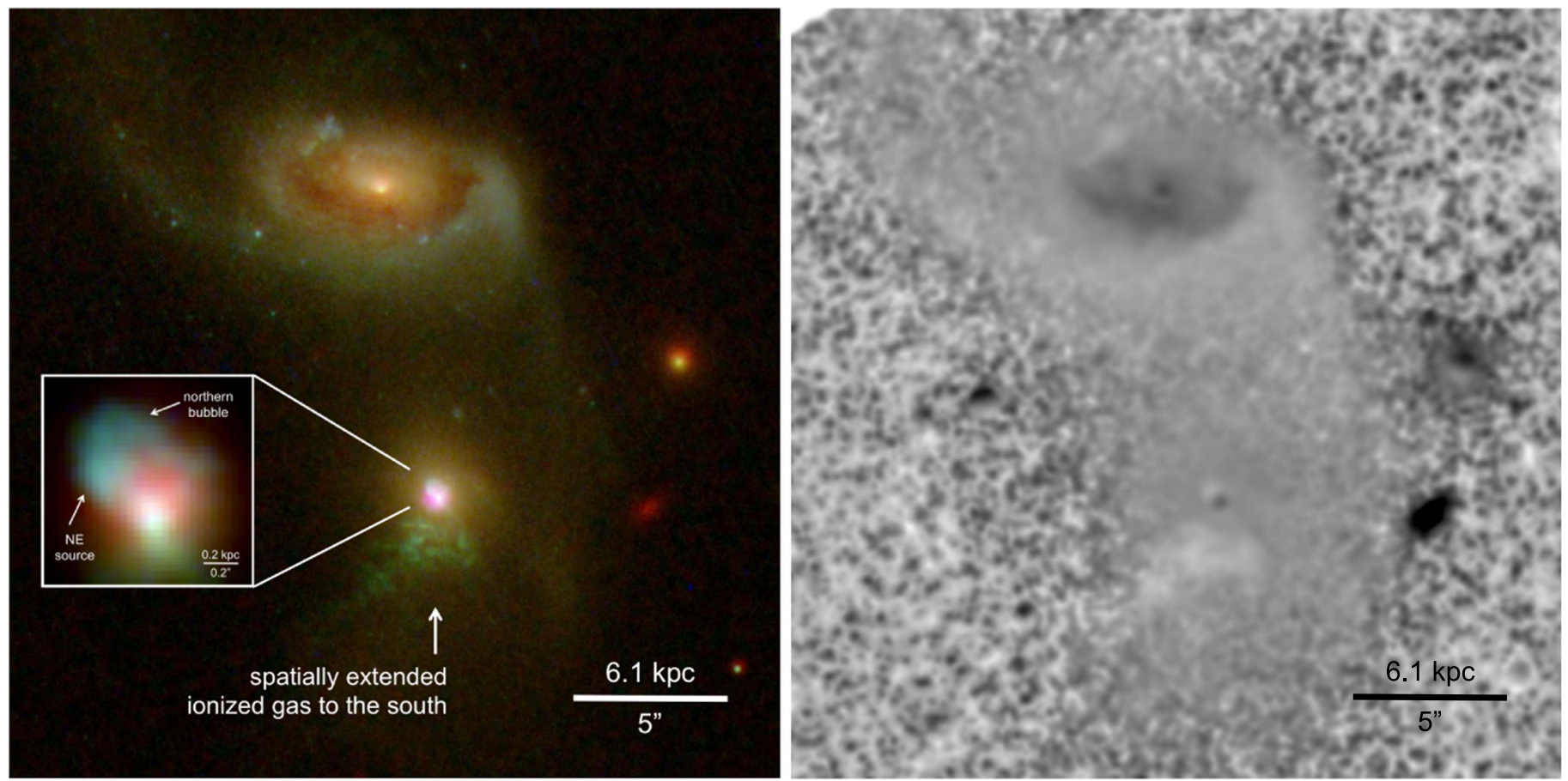

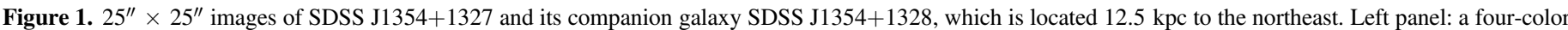

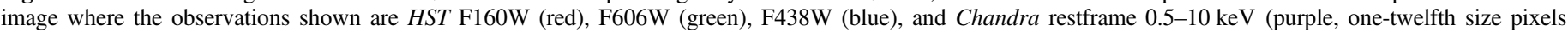

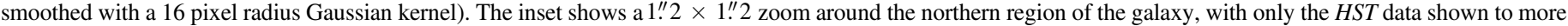

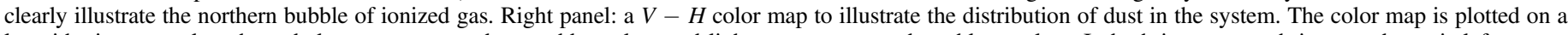
logarithmic grayscale, where dark gray corresponds to redder colors and light gray corresponds to bluer colors. In both images, north is up and east is left.

\section{Observations and Analysis}

\subsection{Optical SDSS Observations}

The spectrum of SDSS J1354+1327 is classified as a Type 2 AGN and it has no evidence of broad emission lines (Oh et al. 2015). We obtain the fluxes of the emission lines in the SDSS spectrum of the galaxy using the OSSY catalog (Oh et al. 2011), which simultaneously fits an entire spectrum using stellar templates for the stellar kinematics and Gaussian templates for the emission components. The host galaxy redshift, based on the template fit to the stellar absorption features, is $z=0.063316 \pm 0.000070$, which we use to convert the [O III] $\lambda 5007$ flux to a luminosity $L_{[\mathrm{O} \text { III] } \lambda 5007}=4.56 \times 10^{41} \mathrm{erg} \mathrm{s}^{-1}$. The $[\mathrm{O} \mathrm{III]}] \lambda 5007$ emission line is redshifted by $69.1 \pm 15.0 \mathrm{~km} \mathrm{~s}^{-1}$ relative to the systemic velocity of the galaxy's stars (Comerford \& Greene 2014). We find that the line flux ratios are $\log ([\mathrm{O} \mathrm{III}] \lambda 5007 / \mathrm{H} \beta)=1.03 \pm 0.05$ and $\log ([\mathrm{N} \mathrm{II}] / \mathrm{H} \alpha)=-0.50 \pm 0.05$, which place SDSS J1354 +1327 in the Seyfert region of the Baldwin-Phillips-Terlevich (BPT) diagram (Baldwin et al. 1981; Kewley et al. 2006).

The companion galaxy, SDSS J1354+1328, has a redshift of $z=0.063586 \pm 0.000048$ and an [O III] $\lambda 5007$ luminosity of $L_{[\mathrm{O} \text { III] } \lambda 5007}=2.4 \times 10^{40} \mathrm{erg} \mathrm{s}^{-1}$. The line flux ratios are $\log ([\mathrm{O} \mathrm{III}] \lambda 5007 / \mathrm{H} \beta)=0.44 \pm 0.09$ and $\log ([\mathrm{N} \mathrm{II}] / \mathrm{H} \alpha)=$ $-0.26 \pm 0.08$, which are in the Seyfert region of the BPT diagram.

\subsection{Chandra/ACIS X-Ray Observations}

SDSS J1354+1327 was observed with Chandra/ACIS for $9443 \mathrm{~s}$ on UT 2014 June 25 as part of the program GO415113X (PI: Comerford). The data were taken with the telescope aimpoint on the ACIS S3 chip in "timed exposure" mode and telemetered to the ground in "faint" mode. We reduced the data with the latest Chandra software (CIAO 4.6.1) and the most recent set of calibration files (CALDB 4.6.2).

We used dmcopy to make sky images of the field in the soft $(S$, restframe $0.5-2 \mathrm{keV})$, hard $(H$, restframe $2-10 \mathrm{keV})$, and total ( $T$, restframe $0.5-10 \mathrm{keV}$ ) energy ranges with pixels binned to $1 / 10$ the native pixel size. Then, we used Sherpa to model the X-ray source as a 2D Lorenztian function in beta $2 \mathrm{~d}$. Our model also included a background component of fixed count rate, which we determined from an adjacent circular region of $30^{\prime \prime}$ radius. We confirmed that the background region does not contain any sources detected by wavdetect with a threshold of sigthresh $=10^{-8}$. We set the initial position of the beta2d component to the location of the SDSS galaxy coordinates. After using psfSize to estimate that the radius of the point spread function (PSF) is 1."07, we ran the fit in Sherpa and allowed the model to fit a region around the galaxy coordinates of three times the PSF size at that location on the chip. We determined the best-fit model parameters by minimizing the Cash statistic using Sherpa's implementation of the "Simplex" minimization algorithm (Lagarias et al. 1998). To test for additional sources, we also attempted fitting a twocomponent beta2d model. However, the amplitude of any second component is detected at $<1 \sigma$ significance, and therefore we adopt the single beta $2 \mathrm{~d}$ model.

To determine the relative positions of the Chandra sources and the HST sources, we registered the Chandra and HST/ F160W images and estimated the relative astrometric uncertainty between the two images. Due to the small number of Chandra sources and the relatively small $H S T / \mathrm{F} 160 \mathrm{~W}$ field of view, we could not directly register the two images. Instead, we registered both images with external images: SDSS $(u, g, r, i$, and $z$ ) and the 2MASS point source catalog (Cutri et al. 2003). This provided us with six independent estimates of the 

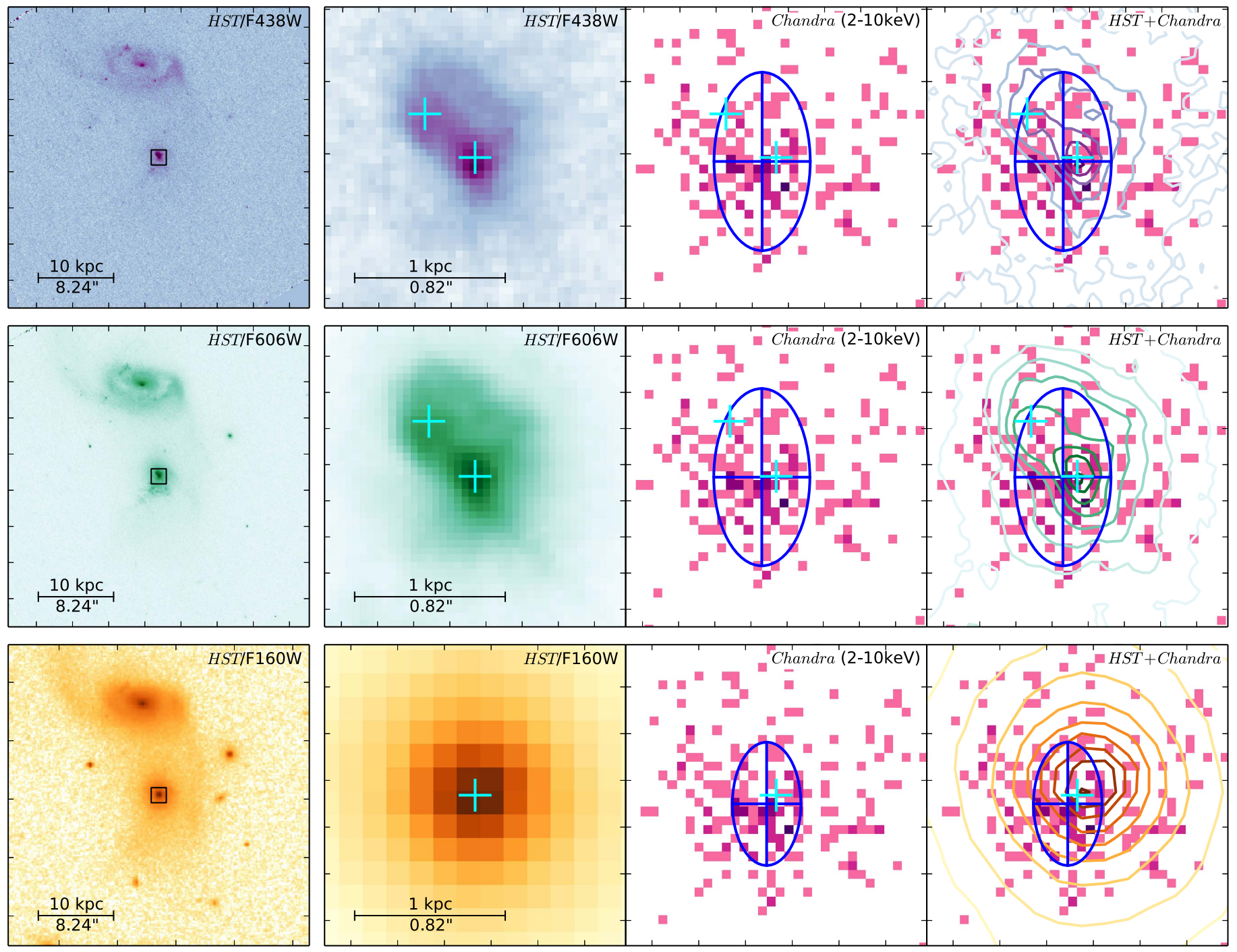

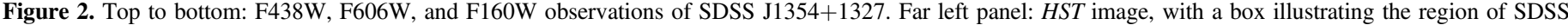

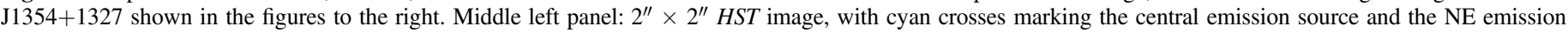

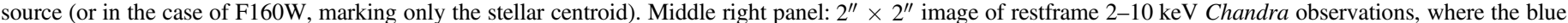

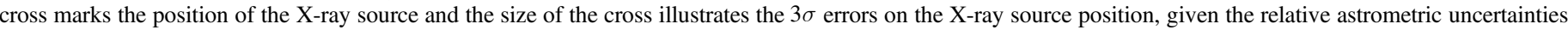

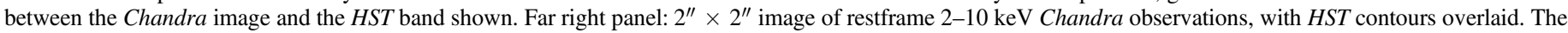
cyan and blue crosses are as shown in the middle left and middle right figures, respectively. In all panels, north is up and east is left.

transformations and relative astrometric uncertainty between the Chandra and HST images. Finally, to minimize the relative astrometric uncertainty, we computed an error-weighted average of the six independent transformations. In the Chandra physical $(X, Y)$ coordinate system, the error-weighted averages of these six transformations yield the final astrometric errors $\overline{\Delta X}=0 . \prime 0583$ and $\overline{\Delta Y}=0 . ! 1104$.

The best-fit positions of the soft, hard, and total X-ray sources, with astrometric shifts applied to align the positions in the HST coordinate frame, are (13:54:29.052, +13:27:56.70), (13:54:29.050, $+13: 27: 56.81$ ), and (13:54:29.047, +13:27:56.84), respectively. The errors on the positions are $\left(00^{\prime \prime} 19,0{ }^{\prime \prime} 50\right),\left(0{ }^{\prime \prime} 07,0{ }^{\prime \prime} 27\right)$, and $(0$. . $06,0.20)$, respectively. The X-ray positions are shown in Figure 2.

Next, we measured the numbers of soft, hard, and total counts attributed to the X-ray source using the Bayesian Estimation of Hardness Ratios (BEHR) code described in (Park et al. 2006). BEHR takes as input the observed soft and hard counts from both the source region and a background region (which we measured using calc-data -sum). The code then uses a Bayesian approach to estimate the expected values and uncertainties of the soft counts, hard counts, total counts, and hardness ratio. Using this approach, we found $S=13.7_{-4.2}^{+3.1}$ counts, $H=238_{-17.0}^{+13.9}$ counts, and $T=252_{-17.0}^{+14.8}$ counts.

We then used Sherpa to model the unbinned energy spectrum of the extracted region over the observed energy range of $2-8 \mathrm{keV}$. For our purposes, we are interested in the observed and intrinsic fluxes integrated over restframe soft, hard, and total energy ranges. Therefore, we fit the spectrum with a redshifted power law, $F=E^{-\Gamma}$, (intended to represent the intrinsic AGN X-ray emission at the SDSS spectroscopic redshift, $\left.z_{\mathrm{SDSS}}\right)$. We also included two multiplicative model components of absorbing neutral hydrogen column densities. The first absorbing component is fixed to the Galactic value, $n_{H, \text { gal }}$, which we determined using an all-sky interpolation of the neutral Hydrogen in the Galaxy (Dickey \& Lockman 1990). 


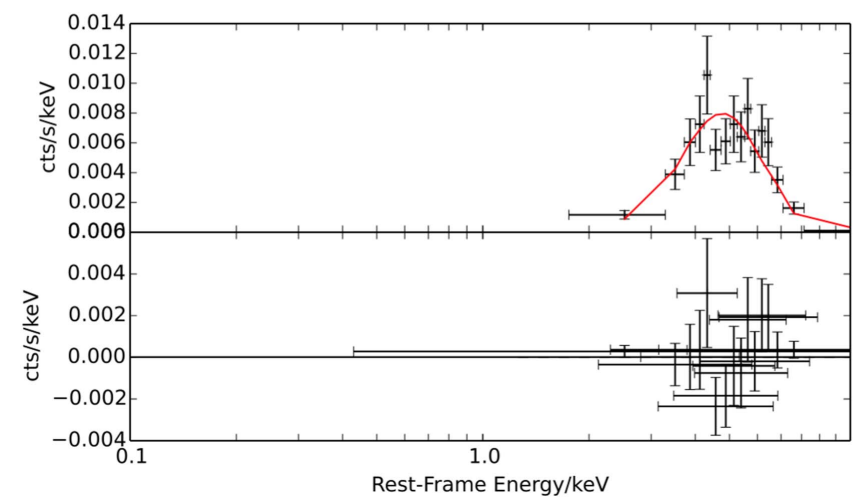

Figure 3. X-ray spectral fit to SDSS J1354+1327, where the top panel shows the fit in red and the bottom panel shows the residuals.

The second absorbing component is allowed to vary and assumed to be intrinsic to the source, $n_{H, \text { exgal }}$, at the redshift $z_{\text {SDSS }}$. All fluxes are $k$-corrected, with the observed values calculated from the model sum (including the absorbing components), while the intrinsic values are calculated from the unabsorbed power-law component. Because we have used Cash statistics, we did not subtract the background before modeling, but we have confirmed that the counts over the 2-8 keV range are dominated by the source region, with a negligible contribution from the background region.

For our first fit to the spectrum, we allowed $\Gamma$ and $n_{H \text {,exgal }}$ to vary freely. We found a best-fit value of $\Gamma=-1.81$ (and a corresponding upper limit on the column density of $3.4 \times$ $10^{21} \mathrm{~cm}^{-2}$ ), which is not within the typical range of observed power-law indices for AGNs, i.e., $1 \leqslant \Gamma \leqslant 3$ (Nandra \& Pounds 1994; Reeves \& Turner 2000; Piconcelli et al. 2005; Ishibashi \& Courvoisier 2010). Consequently, we fixed $\Gamma$ at a value of 1.70 and ran the fit again. We obtained the best-fit model parameters for each combination of parameters by minimizing the Cash statistic using Sherpa's implementation of the Levenberg-Marquardt optimization method (Bevington 1969). The best-fit column density is $1.96_{-0.23}^{+0.29} \times$ $10^{23} \mathrm{~cm}^{-2}$, where the errors do not account for the uncertainty in $\Gamma$, and the reduced Cash statistic is 0.94. Figure 3 shows the best fit to the spectrum.

The restframe hard absorbed (unabsorbed) X-ray fluxes are $1.1_{-0.1}^{+0.2} \times 10^{-12} \mathrm{erg} \mathrm{s}^{-1} \mathrm{~cm}^{-2}\left(2.8_{-0.3}^{+0.3} \times 10^{-12} \mathrm{erg} \mathrm{s}^{-1} \mathrm{~cm}^{-2}\right)$ for $2-10 \mathrm{keV}$. We then used the redshift $z_{\text {SDSS }}$ to convert the X-ray fluxes to X-ray luminosities. The restframe hard absorbed (unabsorbed) luminosities are $L_{\mathrm{X}, 2-10 \mathrm{keV}}=1.1_{-0.1}^{+0.2} \times$ $10^{43} \mathrm{erg} \mathrm{s}^{-1}\left(2.7_{-0.3}^{+0.3} \times 10^{43} \mathrm{erg} \mathrm{s}^{-1}\right)$.

Finally, we searched for an X-ray source at the center of the companion galaxy SDSS J1354+1328, but found none (detection of $0.001_{-0.001}^{+0.002}$ counts in the restframe $0.5-10 \mathrm{keV}$ energy range).

\subsection{HST/WFC3 F160W, F606W, and F438W Observations}

SDSS J1354+1327 was also observed with HST/WFC3 on UT 2014 April 28 (GO 13513, PI:Comerford), and the observations covered three bands: UVIS/F438W ( $B$ band, $954 \mathrm{~s}$ ), UVIS/F606W ( $V$ band, $900 \mathrm{~s}$ ), and IR/F160W ( $H$ band, $147 \mathrm{~s}$ ). The $H S T$ field of view included SDSS $\mathrm{J} 1354+1328$, which is the companion galaxy to the northeast of SDSS J1354+1327. We used GALFIT V3.0 (Peng et al. 2010) to model the light profiles of these two galaxies.
GALFIT is capable of decomposing images of galaxies into multiple components, including a Sérsic profile (e.g., a galactic stellar bulge), exponential disk (e.g., a galactic disk), and an image PSF for bright unresolved sources (e.g., AGNs).

We fit a Sérsic profile (plus a fixed, uniform sky component) to locate the position of each central stellar bulge, as Sérsic profiles have been empirically shown to be good approximations of the light profiles of stellar bulges (Graham \& Driver 2005). To avoid modeling complex and irregular gas kinematics, we only ran GALFIT models on the F160W image because it does not contain significant line emission from ionized gas and it is sensitive to the central stellar bulge, which is the primary component of interest. As shown in Comerford et al. (2015), an additional unresolved point source is not necessary in this model because the AGN contributes negligibly to the F160W flux. The fit was run on a square region of projected physical size $40 \mathrm{kpc}$ on each side to explore within $20 \mathrm{kpc}$ of the AGN position.

We find that the position of SDSS J1354+1327's central stellar bulge is (13:54:29.045, +13:27:56.88), with errors of $(0$. "06, 0." 11). The separation between SDSS J1354+1327 and the companion galaxy is $12.5 \mathrm{kpc}(10$ !' 2$)$. Using the results of the best-fit GALFIT model to each galaxy, we also find the $H$-band luminosity for SDSS J1354+1327 $\left(L_{H}=4.0 \times\right.$ $\left.10^{10} L_{\odot}\right)$ and for the companion galaxy $\left(L_{H}=4.1 \times 10^{10} L_{\odot}\right)$.

To explore the distribution of dust in the galaxy, we made a $V-H$ color map (Martini et al. 2003). We convolved the $V$ and $H$ images with a Gaussian kernel to put them at the same angular resolution, and then computed the magnitude difference $V-H$. The resultant color map is shown in Figure 1 (right). We find that the center of SDSS J1354+1327 is the most obscured part of the galaxy $(V-H=2)$.

Next, we turn our attention to the F438W and F606W observations, which cover a wavelength range that includes $H \delta, H \gamma$, and [O III] $\lambda 4363(\mathrm{~F} 438 \mathrm{~W})$ and $\mathrm{H} \beta$, [O III] $\lambda \lambda$ 4959,5007, [O I] $\lambda \lambda 6300,6363$, [N II] $\lambda \lambda$ 6548,6583, and $\mathrm{H} \alpha$ (F606W). The F438W and F606W observations are dominated by ionized gas emission and not stellar emission, as the optical longslit observations show this to be the case for these wavelength ranges and since the morphologies of the F438W and F606W emission are different from the morphology of the stellar continuum (traced in the F160W observations). We identify two main structures in the F438W and F606W data:

Spatially extended ionized gas to the south. South of the galaxy center is a cone of clumpy, ionized gas (Figure 1, left panel). We map the gas detected in F606W at $>10 \sigma$ above the background, and we find that it is distributed in a cone-like structure with an opening angle of $67^{\circ}$. The gas detected at $>3 \sigma$ then extends $\sim 10 \mathrm{kpc}$ radially from the galaxy center. The range of colors in the cone is $0.2<V-H<1.2$, and the patchiness of the color indicates that in some regions the far side of the cone may be obscured. We explore this in more detail in Section 3.2.

Northern bubble. North of the galaxy center is a loop of emission that extends to a maximum distance of 0. " $6(0.7 \mathrm{kpc})$ from the galaxy center. The bubble's color is $V-H=0.7$. By fitting a Sérsic component, we find that the brightest component of this bubble is located northeast of the galaxy center. We call this feature the NE source, and in F606W its apparent magnitude is 21.4 (compared with an apparent magnitude of 18.7 for the galaxy). The positions of the NE source in F438W (13:54:29.064, +13:27:57.10) and F606W 

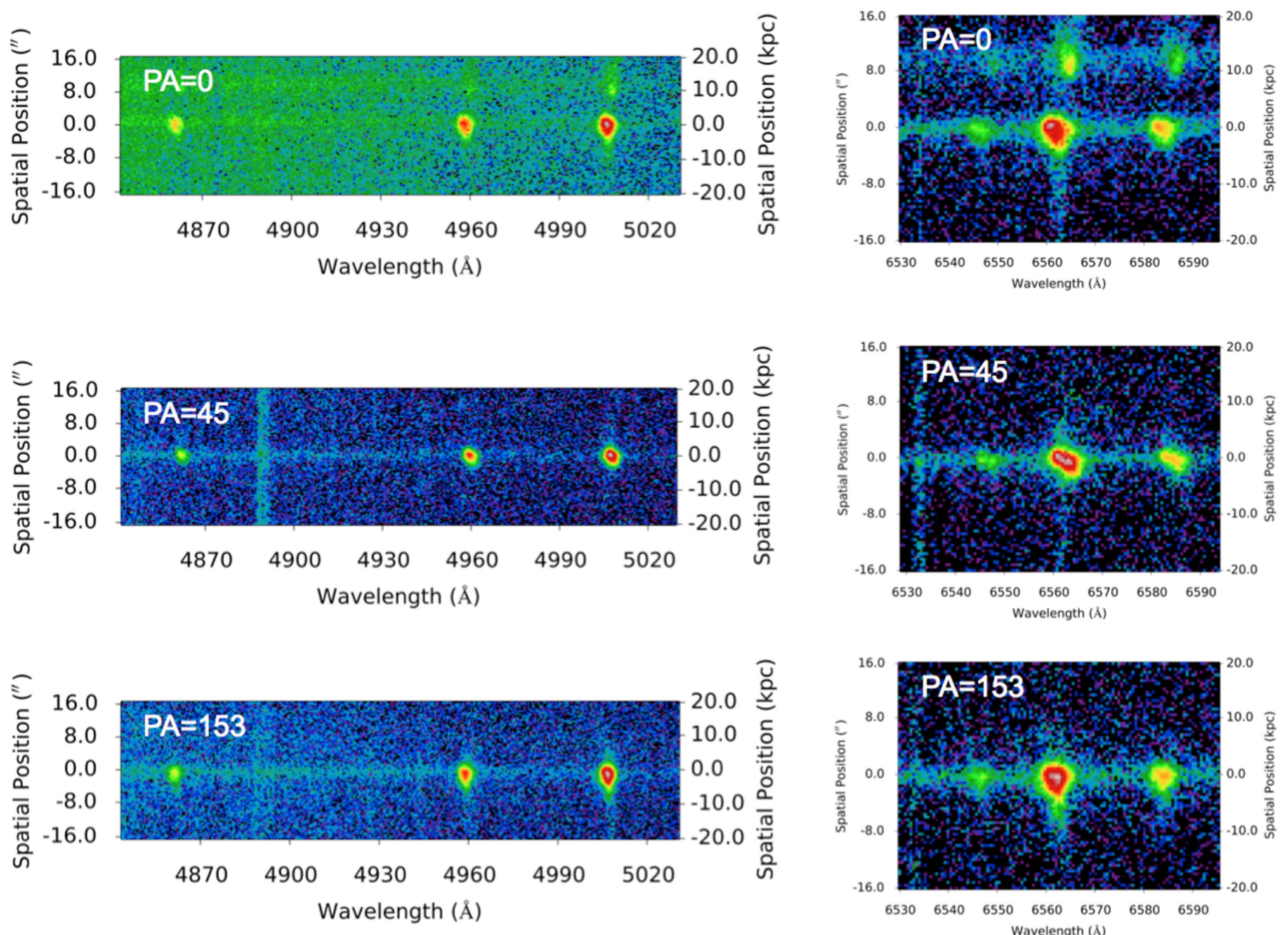

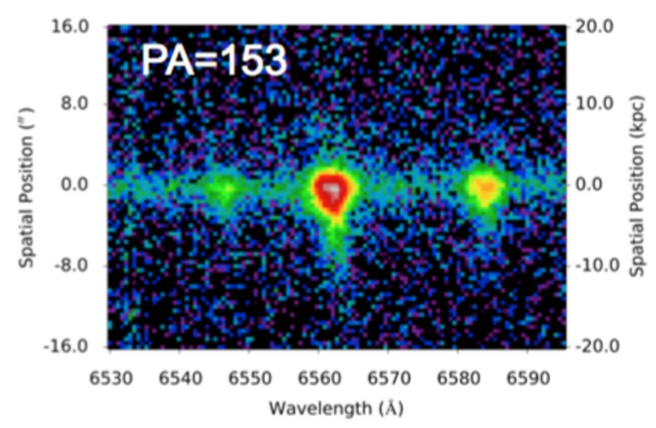

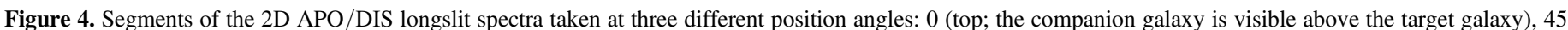

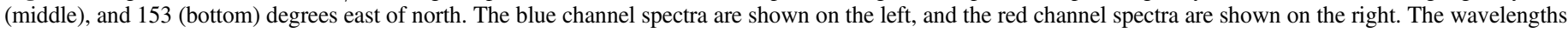

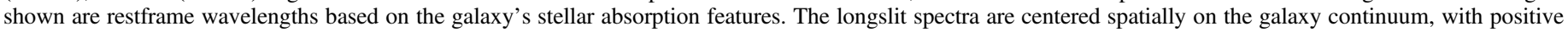
spatial positions to the north and negative spatial positions to the south.

$(13: 54: 29.062,+13: 27: 57.10)$ agree to within $0 . " 04$, so we take the NE source position to be the average of the positions measured in F438W and F606W. The NE source is located 0 "' $33(0.40 \mathrm{kpc})$ from the galaxy center.

\subsection{APO/DIS Optical Longslit Observations}

We observed SDSS J1354+1327 with APO/DIS on UT 2015 December 18 and UT 2016 June 30. We used a 1200 lines $\mathrm{mm}^{-1}$ grating, centered so that the wavelength range covered $\mathrm{H} \beta$ and [O III] on the blue channel and [N II] and $\mathrm{H} \alpha$ on the red channel. We used a 1". 5 slit width, and the DIS pixel scales are 0 ." 42 on the blue channel and $0 . " 40$ on the red channel. Following the approach used to study active galaxies with double-peaked narrow emission lines (Comerford et al. 2012; Nevin et al. 2016), we observed SDSS J1354+1327 at multiple different slit position angles to understand the kinematics of the features of the galaxy that are seen in the HST images. We observed the galaxy at three different slit position angles (given in degrees East of North): $0^{\circ}$ to cover the emission seen in the north-south direction and the companion galaxy to the north, $153^{\circ}$ to cover the bright clumps of emission seen to the south of the galaxy center, and $45^{\circ}$ to cover the NE emission source. We observed for $1200 \mathrm{~s}$ at each position angle, and the data were reduced using standard IRAF procedures.

The longslit spectra are shown in Figure 4. We fit Gaussians to the [O III] $\lambda 5007$ and $\mathrm{H} \alpha$ flux at each position along the slit where the emission line is detected with a signal-to-noise ratio $(\mathrm{S} / \mathrm{N})>10$, and we extracted the line-of-sight velocities. These are shown in Figure 5.

Next, we identify where the peak of [O III] $\lambda 5007$ flux is located. The longslit orientation of position angle 45 has the strongest [O III] $\lambda 5007$ flux (integrated flux of $2.6 \times 10^{-13}$ erg s${ }^{-1} \mathrm{~cm}^{-2}$, which is twice the integrated flux at position angle 0 and 2.4 times the integrated flux at position angle 153), and we fit a Gaussian to the spatial distribution of [O III] $\lambda 5007$ flux along this slit position angle. We find that the peak flux of [O III] $\lambda 5007$ occurs 0 ." $41 \pm 0$." 08 to the north of the galaxy along position angle 45 , which is spatially coincident with the NE source seen in the HST images. The velocity of the $[\mathrm{O}$ III $] \lambda 5007$ peak flux, relative to systemic, is $66.8 \pm 11.5 \mathrm{~km} \mathrm{~s}^{-1}$. This explains the velocity offset of $69.1 \pm 15.0 \mathrm{~km} \mathrm{~s}^{-1}$ seen in the SDSS spectrum of SDSS $\mathrm{J} 1354+1327$. 


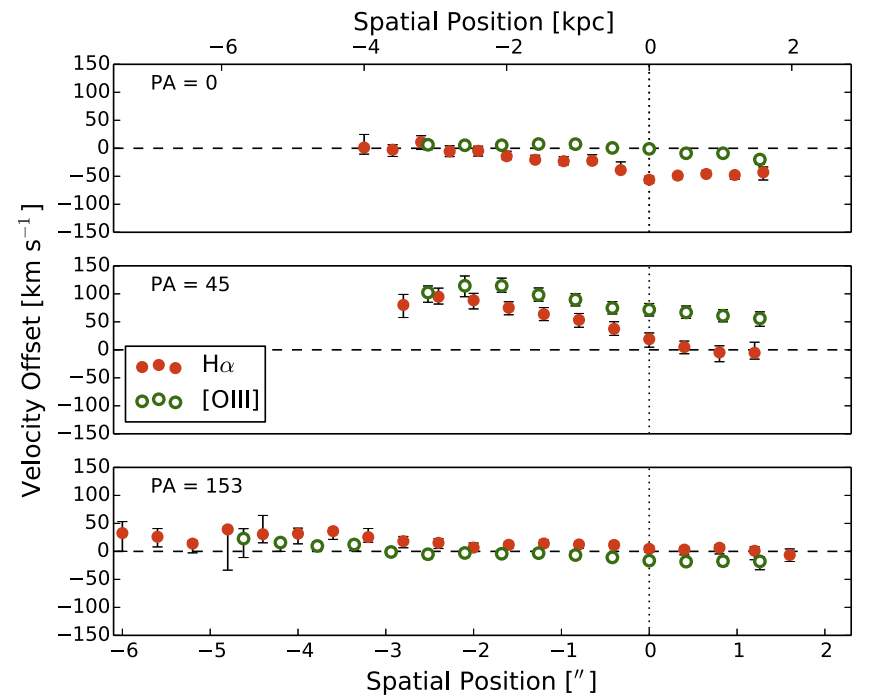

Figure 5. Line-of-sight velocity offsets of emission lines along the three different position angles used for the APO/DIS longslit observations. $\mathrm{H} \alpha$ is illustrated with filled red circles, while $[\mathrm{O}$ III] $\lambda 5007$ is illustrated with open green circles, and along each slit the emission detected with $\mathrm{S} / \mathrm{N}$ greater than 10 is shown. A velocity offset of zero (dashed horizontal line) is the systemic velocity of the galaxy's stars. The spatial position zero marks the centroid of the galaxy continuum (vertical dotted line), with positive spatial positions to the north and negative spatial positions to the south.

We then use emission line flux ratios in the BPT diagram (Baldwin et al. 1981; Veilleux \& Osterbrock 1987; Kewley et al. 2006) to identify the sources of the ionized emission in SDSS J1354+1327. We measure these line flux ratios at each position along the slit where the $\mathrm{S} / \mathrm{N}$ is $>3$ for each of the four emission lines, and the results are shown in Figure 6. At all observed position angles, the ionized gas has Seyfert-like or composite line ratios.

To probe the relative roles of photoionization and shocks in the galaxy, we measure the line flux ratios [O I] $\lambda 6300 / \mathrm{H} \alpha$ and He II $\lambda 4686 / \mathrm{H} \beta$ at each position along the slit where the $\mathrm{S} / \mathrm{N}$ is $>3$ for each of the four emission lines. These line flux ratios discriminate between AGN photoionization and shock models (e.g., Clark et al. 1997; Moy \& Rocca-Volmerange 2002), and we have illustrated these models in Figure 7. The photoionization models are computed with CLOUDY (Ferland 1996) and the shock models are computed with MAPPINGSIII (Dopita \& Sutherland 1996).

To explore the densities of the material within the galaxy, we also measure the line flux ratio [S II] $\lambda \lambda 6717 / 6731$ at each position along the slit where the $S / N$ is $>3$ for both emission lines. Then, we convert this to an electron density (Osterbrock \& Ferland 2006; Schnorr-Müller et al. 2016) as shown in Figure 8 . We find that the electron densities are consistent with those of a typical narrow-line region $\left(10^{2} \lesssim n_{\mathrm{e}}\left(\mathrm{cm}^{-3}\right) \lesssim 10^{3}\right.$; Osterbrock \& Ferland 2006), and that the electron density is higher south of the galaxy center and lower north of the galaxy center.

From the longslit spectra, we identify three main kinematic components in the galaxy:

Rotating disk. The rotating disk is most apparent at position angle 45 (Figure 5, middle), where the velocities progress from redshifts to the south to blueshifts to the north. We find that the $\mathrm{H} \alpha$ emission is best fit by a rotating disk that has a position angle on the sky of $53^{\circ}$ East of North, is inclined $50^{\circ}$ into the plane of the sky, has an intrinsic velocity of $182 \mathrm{~km} \mathrm{~s}^{-1}$, has a radius of $2.5 \mathrm{kpc}$, and whose kinematic center is located at the galaxy's stellar centroid. This rotating disk is also seen in $\mathrm{Pa} \alpha$, with the same disk parameters.

Spatially extended ionized gas to the south. South of the galaxy center, we find ionized gas that extends to distances $\gtrsim 4 \mathrm{kpc}$ from the galaxy center. We focus our analysis on [O III] $\lambda 5007$ because it is the strongest emission line and because $\mathrm{H} \alpha$ is contaminated by the disk rotation. We find that the $[\mathrm{O} \mathrm{III}] \lambda 5007$ velocities south of the galaxy center range from $24 \mathrm{~km} \mathrm{~s}^{-1}$ redshifted relative to systemic (along position angle 153) to $20 \mathrm{~km} \mathrm{~s}^{-1}$ blueshifted relative to systemic (along position angle 153; Figure 5). The line flux ratios indicate a photoionized origin for emission south of the galaxy center (Figure 7).

Northern bubble. North of the galaxy center is ionized gas that extends $\sim 1 \mathrm{kpc}$ from the galaxy center. The [O III] $\lambda 5007$ velocities north of the galaxy center range from $72 \mathrm{~km} \mathrm{~s}^{-1}$ redshifted relative to systemic (along position angle 45) to $20 \mathrm{~km} \mathrm{~s}^{-1}$ blueshifted relative to systemic (along position angle 0; Figure 5). The [O III] $\lambda 5007$ emission is strongest along position angle 45 , which is the position angle aligned with the brightest component of the northern bubble seen in the HST observations. The line flux ratios at the position of the NE source are inconsistent with pure photoionization models, and the high [He II] $\lambda 4686 / \mathrm{H} \beta$ ratio can instead be explained by a shock with a precursor H II region (e.g., Sutherland et al. 1993); Moy \& Rocca-Volmerange (2002) find regions of AGN emission with similar line flux ratios and interpret them also as produced by a shock with a precursor.

\subsection{Keck/OSIRIS Near-infrared IFS Observations}

We observed SDSS J1354+1327 on UT 2013 April 3 using Laser Guide Star Adaptive Optics (LGS-AO) with Keck/ OSIRIS IFS. We used the Kn1 filter with the 0 " 1 pixel scale and the associated 3". $6 \times 6$ ". 4 field of view to ensure that the observations would cover the entire area of a $3^{\prime \prime}$ diameter SDSS fiber. The observations covered an observed wavelength range of $1.955-2.055 \mu \mathrm{m}$ and detected the $\operatorname{Pa} \alpha 1.87 \mu \mathrm{m}$ emission line, which traces the spatial distribution and kinematics of the ionized gas in the galaxy. We used the galaxy nucleus as the tip-tilt star, observed at a position angle of 0 degrees East of North, and integrated for 30 minutes. The data were reduced with the OSIRIS data reduction pipeline, and we used the IDL code LINEFIT (Davies et al. 2007) to create 2D kinematic and flux distribution maps (Figure 9).

From the OSIRIS observations, we identify three main kinematic components in the galaxy:

Rotating disk. To model the rotating disk, we first mask the pixels in the $\mathrm{Pa} \alpha$ velocity map that have a flux density lower than $10 \%$ of the peak of the continuum emission. We then use the KINEMETRY code (Krajnović et al. 2006) to model the rotating disk structure seen extending from the northeast (blueshifted) to the southwest (redshifted) in $\mathrm{Pa} \alpha$. The parameters of the best-fit model are a position angle of 55 degrees east of north, an inclination of 53 degrees, and a kinematic center that is spatially offset by $0 . ! 2$ northeast of the peak of the stellar continuum. The parameters of the rotating disk observed in $\mathrm{Pa} \alpha$ are consistent with those of the rotating disk observed in the optical longslit spectra. After the disk model is subtracted from the data, we examine the velocity residuals, which have $\pm 20 \mathrm{~km} \mathrm{~s}^{-1}$ errors (Figure 9, bottom right panel). The velocity residuals are not significant enough 

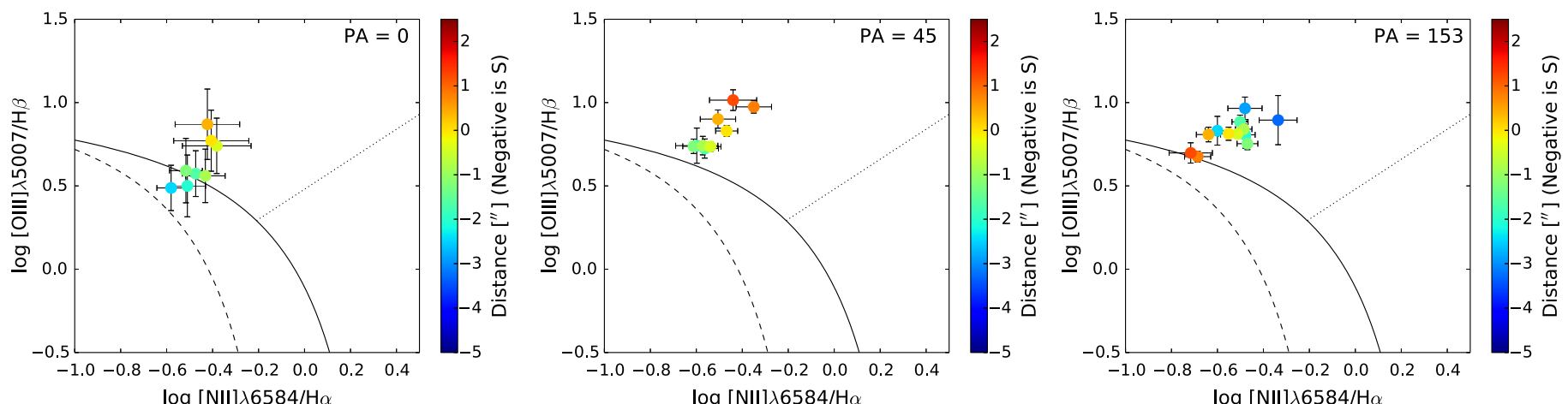

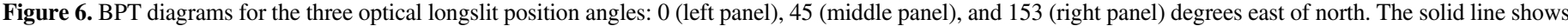

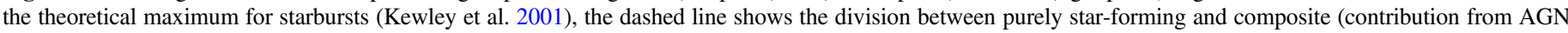

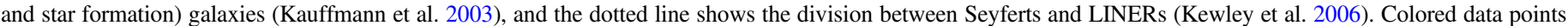
and $1 \sigma$ error bars are plotted for each position along the slit, where zero is defined as the position of the host galaxy continuum on the slit.

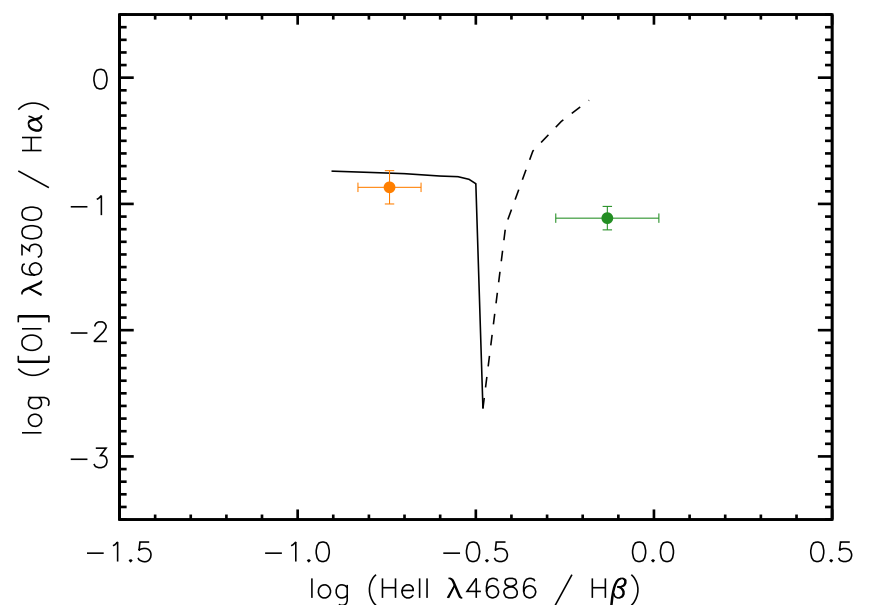

Figure 7. [O I] $\lambda 6300 / \mathrm{H} \alpha$ vs. [He II] $\lambda 4686 / \mathrm{H} \beta$. The orange point illustrates the line flux ratios of the emission located $3 \mathrm{kpc}$ south of the galaxy center along position angle $153^{\circ}$, which is part of the spatially extended ionized gas to the south. The green point illustrates the line flux ratios of the emission located $0.5 \mathrm{kpc}$ north of the galaxy center along position angle $45^{\circ}$, at the location of the NE source. The solid line represents pure photoionization models with ionization parameter $U$ varying between $\log U=-1$ (bottom) and $\log U=-4$ (top), and a spectral index of the ionizing continuum $\alpha=-1$. The dashed line represents a model of shocks and a matter bound precursor, where the shock has $v=1000 \mathrm{~km} \mathrm{~s}^{-1}$ (models adapted from Moy \& Rocca-Volmerange 2002).

to fit a secondary component. We find that the kinematic center of the rotating disk is offset by $0.12(0.3 \mathrm{kpc})$ from the stellar continuum center, and we attribute this perturbation to a recent pericenter passage and interaction with the companion galaxy.

Spatially extended ionized gas to the south. While the OSIRIS field of view is dominated by the rotating disk, there is also extraplanar $\mathrm{Pa} \alpha$ emission visible to the south of the galaxy center, and this emission is detected at $5 \sigma$ significance out to the edge of the field of view (Figure 9, top middle). The line-ofsight velocities of the gas are $\pm 30 \mathrm{~km} \mathrm{~s}^{-1}$. The Pa $\alpha$ emission also follows the conical structure seen in the HST observations.

Northern bubble. There is spatially extended $\mathrm{Pa} \alpha$ emission associated with the loop seen in the HST data.

\section{Results}

\subsection{Nature of the X-Ray Source: A Central AGN}

The X-ray source in SDSS J1354+1327 has an observed luminosity of $L_{\mathrm{X}, 2-10 \mathrm{keV}}=1.1 \times 10^{43} \mathrm{erg} \mathrm{s}^{-1}$, which is brighter than the typical AGN luminosity threshold of

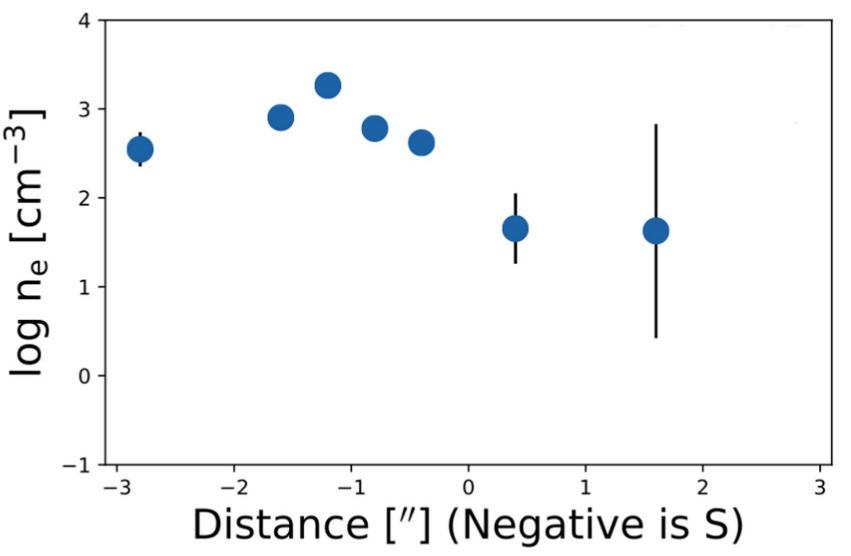

Figure 8. Electron density measured along the APO/DIS longslit spectrum taken at position angle $45^{\circ}$ east of north. Zero is defined as the position of the host galaxy continuum on the slit, positive spatial positions are to the north, and negative spatial positions are to the south. Along this position angle, the NE source is located 0." 41 to the north (this position corresponds with the first data point north of the continuum), and the electron density is an order of magnitude lower at the NE source than at the southern positions on the slit.

$L_{\mathrm{X}, 2-10 \mathrm{keV}}>10^{42} \mathrm{erg} \mathrm{s}^{-1}$ and shows that the X-ray source is associated with an AGN. Mid-infrared colors from the Widefield Infrared Survey Explorer (WISE) also show that an AGN is present (SDSS J1354+1327 has $W 1-W 2=1.14 \pm 0.03$, where $W 1-W 2 \geqslant 0.8$ indicates that the mid-infrared flux is dominated by an AGN; Stern et al. 2012).

The fit to the $\mathrm{X}$-ray spectrum reveals a relatively high column density $n_{H \text {,exgal }}=2 \times 10^{23} \mathrm{~cm}^{-2}$, which indicates that the source is an X-ray absorbed AGN. This is consistent with the $V-H$ dust map, which shows that the very center of the galaxy is the most obscured (Figure 1, right panel).

Next, we consider the position of the X-ray source within the host galaxy. We have identified the position of the X-ray source, the positions of the central stellar nucleus of the galaxy and the NE emission source visible in the HST F438W and F606W observations, and performed astrometric corrections. We compare the positions to determine if the X-ray AGN position aligns with any of these galaxy features (Figure 2, right panel). If the $\mathrm{X}$-ray $\mathrm{AGN}$ is associated with the $\mathrm{NE}$ source, then the AGN is located $0.40 \mathrm{kpc}$ from the center of SDSS J1354+1327 and this spatial offset could be explained as an offset AGN, a gravitational recoil AGN, or a gravitational slingshot AGN. We find that the position of the X-ray AGN is more consistent with the position of the stellar centroid of the 

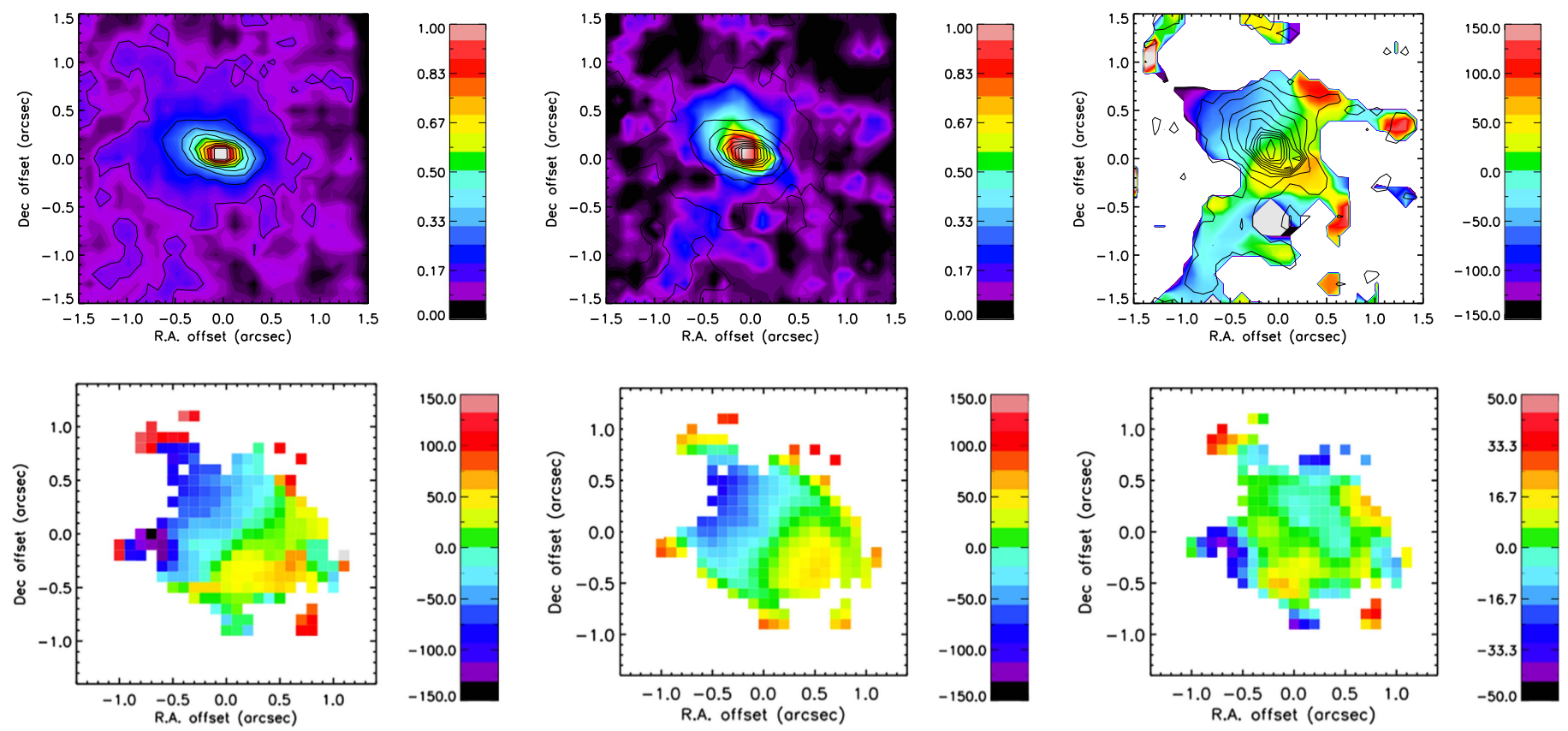

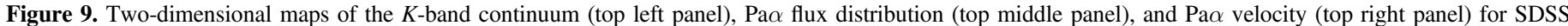

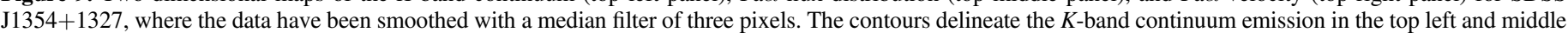

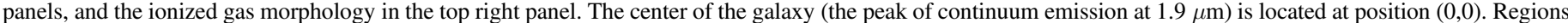

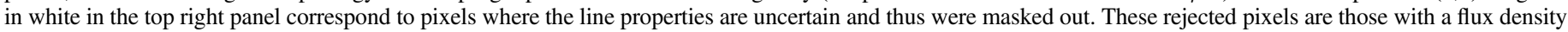

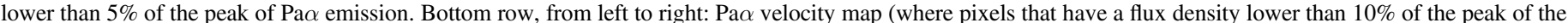

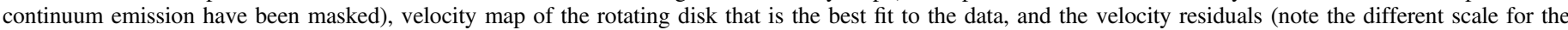
velocity color bar). Each map is centered on the kinematic center of the galaxy. In all maps north is up and east is left.

galaxy (consistent to within $\lesssim 1 \sigma$ ) than with the position of the NE source (consistent to within $\lesssim 2.5 \sigma$ ). Consequently, we conclude that the X-ray AGN is most likely located at the galaxy center.

\subsection{Nature of the Spatially Extended Ionized Gas to the South: A Photoionized AGN Outflow}

There is extraplanar ionized gas extending south of the galaxy center, as seen in the HST F606W and F438W observations, the APO/DIS optical longslit observations, and the Keck/OSIRIS near-infrared IFS observations. The gas is clumpy, as seen morphologically in the HST data (Figure 1, left panel) and in the mixture of redshifted and blueshifted line-ofsight velocities of the emission lines observed with APO/DIS (Figure 5).

Here, we aim to determine whether the gas is inflowing, outflowing, or passively photoionized by the central AGN. If the AGN is passively photoionizing the gas in the galaxy, then there should be symmetric photoionized cones on either side of the AGN due to the collimating torus (e.g., Schmitt et al. 2003), but this is not the case for SDSS J1354+1327. In the passive photoionization scenario, the gas also should be rotating (with possible deviations due to the interaction with the companion galaxy) because it is following the galaxy potential. We fit a rotating disk to the optical and near-infrared spectra, and find that the rotating disk is oriented with a position angle 54 degrees east of north, but no rotation is seen in the gas extending to the south of that disk. Instead, the southern gas has blueshifts and redshifts observed along directions not coincident with the axis of rotation, as can be seen in the velocities in the longslit data. This is evidence of an inflow or outflow, rather than passively photoionized gas.
The morphology of the southern gas distinguishes between the inflow and outflow scenarios. As shown in the HST images (Figure 1, left panel), the gas has a conical morphology with the cone beginning at the galaxy center and extending to the south, outside the plane of rotation of the galaxy. This conical morphology is typical of AGN outflows (e.g., Mulchaey et al. 1996; Schmitt et al. 2003), where the torus provides the collimation (e.g., Antonucci \& Miller 1985; Malkan et al. 1998). In contrast, conical morphologies are not expected for inflows, which are typically radial streamers of gas (e.g., Iono et al. 2004; Müller-Sánchez et al. 2009).

We conclude that the spatially extended ionized gas to the south is an outflow. We apply an analytic Markov Chain Monte Carlo model to the longslit observations to model the data as a biconical outflow, as done for similar longslit observations of galaxies in Nevin et al. (2017). We find that the data are best fit (with a reduced $\chi^{2}=1.5$ ) by a bicone extending south of the galaxy center at an inclination of $27_{-22}^{+14}$ degrees out of the plane of the sky, a position angle of $190_{-25}^{+26}$ degrees east of north on the plane of the sky, a half opening angle of $37_{-15}^{+10}$ degrees, a turnover radius of $1.9_{-1.7}^{+2.4} \mathrm{kpc}$, a maximum velocity at the turnover radius $V_{\max }=40_{-30}^{+40} \mathrm{~km} \mathrm{~s}^{-1}$, and a lateral surface area $A=4_{-4}^{+14} \mathrm{kpc}^{2}$. The observed blueshifted and redshifted velocities of the gas imply that we are seeing parts of the front and rear facing walls of the outflow (e.g., Bae \& Woo 2016), which fits with the clumpiness of the gas and dust partially obscuring the view (Figure 1, right). When we project the cone onto the plane of the sky, we find an observed opening angle of $66_{-27}^{+18}$ degrees, which is consistent with the opening angle of 67 degrees measured from the HST data.

Then, we use the best-fit parameters of the outflow model, our measurement of the electron density $n_{\mathrm{e}}$, and assume a 
filling factor $f=0.01$ to be conservative in our energy calculation to estimate the mass outflow rate $\dot{M}=m_{\mathrm{p}} n_{\mathrm{e}} V_{\mathrm{max}} f A$, where $m_{\mathrm{p}}$ is the proton mass. We find $\dot{M}=6.5_{-6.3}^{+52.3} M_{\odot} \mathrm{yr}^{-1}$.

This outflow could be driven by either star formation or by an AGN. First, we consider the scenario of a star formation driven outflow. To set an upper limit on the star formation rate (SFR) in SDSS J1354+1327, we assume that all of the $\mathrm{H} \alpha$ emission is associated with star formation. Using the luminosity measured from the SDSS spectrum, $L_{\mathrm{H} \alpha}=$ $1.4 \times 10^{41} \mathrm{erg} \mathrm{s}^{-1}$, the SFR from Kennicutt \& Evans (2012) is then $\mathrm{SFR}=5.37 \times 10^{-42} L_{\mathrm{H} \alpha}=0.75 M_{\odot} \mathrm{yr}^{-1}$. When we convert this SFR to a mass outflow rate, we find an upper limit of $\dot{M}_{\mathrm{SF}}=0.2 M_{\odot} \mathrm{yr}^{-1}$ (Veilleux et al. 2005), which is too weak to drive the observed outflow. We also note that a star formation driven outflow that is powered by supernovae in the disk of the galaxy typically has a wide, chimney-shaped morphology of the gas being driven from the disk into the halo (e.g., Norman \& Ikeuchi 1989), which is inconsistent with the conical morphology of the ionized gas observed in SDSS $\mathrm{J} 1354+1327$.

Consequently, we conclude that the outflow is AGN driven. This is consistent with the [O III] $\lambda 5007 / \mathrm{H} \beta$ and $[\mathrm{N} \mathrm{II}] / \mathrm{H} \alpha$ line flux ratios at the location of the outflow, which show that the emission is Seyfert driven. Further, the [O I] $\lambda 6300 / \mathrm{H} \alpha$ and [He II] $\lambda 4686 / \mathrm{H} \beta$ line flux ratios and the conical morphology each indicate that the emission is photoionized (e.g., Wilson \& Tsvetanov 1994). The AGN luminosity is also sufficient for photoionizing the cone of gas, based on photoionization models computed using the spectral synthesis code CLOUDY (Ferland et al. 2013; Richardson et al. 2014). We conclude that the spatially extended ionized gas to the south is a photoionized AGN outflow.

Although we do not know the velocity of the outflow at the time it was launched, we can use the current observed velocity and spatial extent of the outflow to find an upper limit of $10^{8}$ years on the timescale for the outflow to produce the cone of gas. The light-travel timescale plus the recombination timescale $\left(t_{\mathrm{rec}} \approx\left(\alpha n_{\mathrm{e}}\right)^{-1}\right.$, where the recombination coefficient $\alpha=1.72 \times 10^{-11} \mathrm{~cm}^{3} \mathrm{~s}^{-1}$ for [O III] and $n_{\mathrm{e}}$ is from Section 2.4; Osterbrock \& Ferland 2006) to illuminate the gas is then $\sim 50$ years, which is negligible. Given that the emission can be observed for up to $10^{5}$ years (e.g., Schawinski et al. 2015), we conclude that the outflow was likely launched $\lesssim 10^{5}$ years ago.

\subsection{Nature of the Northern Bubble: A Shocked AGN Outflow}

The HST F438W and F606W observations reveal a loop of gas extending $0.7 \mathrm{kpc}$ north of the galaxy center, as well as a $\mathrm{NE}$ source that is located $0.40 \mathrm{kpc}$ northeast of the galaxy center and is embedded in the loop of emission (Figure 1, left panel). The peak of [O III] $\lambda 5007$ emission is located at the NE source as well.

As we did with the spatially extended ionized gas to the south, we consider whether the northern bubble is caused by gas that is inflowing, outflowing, or passively photoionized by the AGN. First, the off-nuclear peak of the [O III] $\lambda 5007$ emission indicates a shock at the position of the NE source and explains the velocity-offset emission line in the SDSS spectrum. We also find that the observed line flux ratios cannot be explained by pure photoionization, and instead are explained by shocks. Further evidence for shocks comes from the looped morphology of the northern bubble, which indicates that shocks are being driven into the ambient gas (e.g., Faucher-Giguère \& Quataert 2012; Gabor \& Bournaud 2014). Where we see redshifted velocities, we are seeing the back side of the bubble.

The presence of shocks rules out the scenario of an AGN passively photoionizing the northern gas in SDSS J1354 +1327 , and leaves inflowing or outflowing gas as the remaining scenarios. Inflowing gas typically streams radially toward the galaxy center (e.g., Iono et al. 2004; Müller-Sánchez et al. 2009), and the bubble morphology of the observed gas is difficult to explain for an inflow. In contrast, many AGN outflows are seen blowing bubbles of gas (e.g., Fabian 2012; Greene et al. 2014). We conclude that the northern bubble is an AGN outflow driving shocks into the ambient material.

The original velocity of the outflow is unknown, but we measured the current observed velocity and spatial extent of the bubble. Using these values, we estimate that the outflow was launched $\lesssim 10^{7}$ years ago. Taking into account the length of time that the emission signatures can linger, the outflow must have been launched $\lesssim 10^{5}$ years ago.

\subsection{Companion Galaxy to SDSS J1354+1327}

SDSS J1354+1327 has a companion galaxy, SDSS $\mathrm{J} 135429.17+132807.3$, which is located $12.5 \mathrm{kpc}$ to the northeast and is redshifted by $76 \mathrm{~km} \mathrm{~s}^{-1}$ relative to SDSS J1354+1327. Emission line diagnostics of the companion's SDSS spectrum suggest that it hosts an AGN, and Liu et al. (2011) also noted that SDSS J1354+1327 and SDSS J1354 +1328 are a pair of active galaxies. However, there is no WISE source detected at the location of the companion galaxy. Further, we do not detect an AGN in our Chandra observations of the companion galaxy.

The lack of an X-ray detection suggests that the AGN is obscured, the AGN's X-ray luminosity is low on the predicted [O III] $\lambda 5007$ to X-ray luminosity scaling relation, some of the gas covered by the SDSS fiber is ionized by SDSS J1354 +1327 's AGN, or that the [O III] $\lambda 5007$ emission is a light echo of past AGN activity (e.g., Lintott et al. 2009; Schawinski et al. 2015; the AGN may be flickering on and off, or the SMBH may have been ejected from the host galaxy center). The other possibility is that the [O III] $\lambda 5007$ luminosity is not associated with an AGN. In this scenario, the observed line flux ratio $(\log ([\mathrm{O} \mathrm{III}] / \mathrm{H} \beta)=0.44)$ could be produced by a starburst instead of an AGN, given that models predict that starbursts can produce line ratios that high. Given that the dust map indicates obscuration at the galaxy center $(V-H=2.4)$, we conclude that the galaxy most likely hosts an obscured AGN.

The HST observations show tidal tails of stars connecting the primary and companion galaxies, indicating that they are already interacting (Figure 2, bottom left panel). Taking the mass ratio to be the luminosity ratio of the two stellar bulges, the merger's mass ratio is 0.98 (SDSS J1354+1327 is slightly less massive).

\section{Nature of SDSS J1354+1327: Episodic AGN Outbursts}

We have shown that SDSS J1354+1327 hosts asymmetric AGN-driven outflows. South of the galaxy center there is a large, $\sim 10 \mathrm{kpc}$ extended outflow of ionized gas, while north of the galaxy center there is a small-scale $(\sim 1 \mathrm{kpc})$ bubble from a 

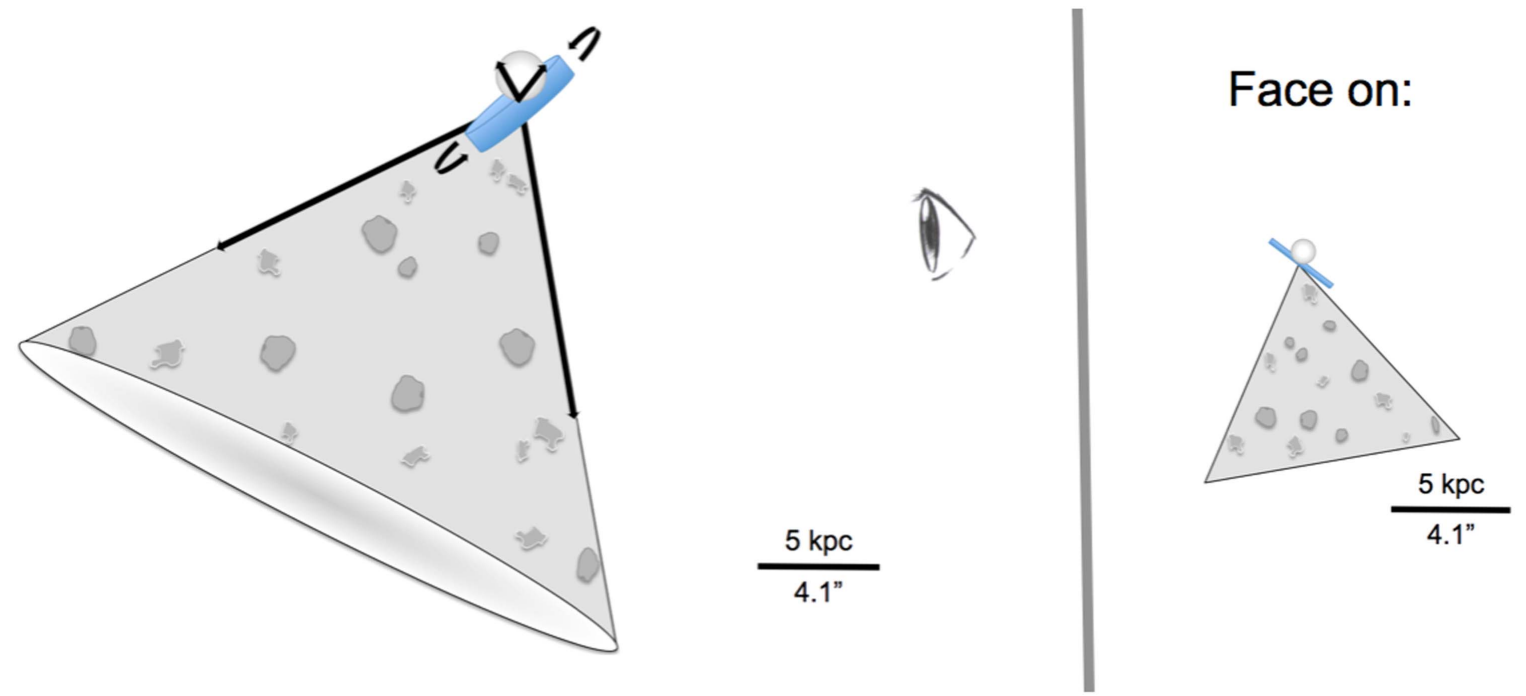

Figure 10. Left panel: 3D drawing of the main components of SDSS J1354+1327: a southern conical outflow (with clumpiness shown), a northern bubble, and a rotating disk. Arrows show the directions of the gas motion in each component, and an eye shows the orientation of the observer. Right panel: face-on view of the galaxy's main components, as shown from the perspective of the observer.

shocked AGN outflow. The main components of SDSS J1354 +1327 are illustrated in a diagram in Figure 10.

One possible explanation for the asymmetry of the outflow is that there were concurrent AGN outbursts to the north and south, but the southern outburst traveled more efficiently while the northern outburst was blocked by high density ISM clouds (e.g., DeGraf et al. 2017). In this scenario, dense clouds to the north of the AGN would absorb the outflow energy and radiate it away, while the southern outflow would propagate unimpeded. However, in SDSS J1354+1327 we find that the density at the northern outburst $\left(n_{\mathrm{e}}=45 \mathrm{~cm}^{-3}\right)$ is an order of magnitude lower than the densities associated with the southern outburst $\left(n_{\mathrm{e}} \geqslant 420 \mathrm{~cm}^{-3}\right)$, which does not support the concurrent AGN outburst scenario.

Another possible explanation for the asymmetric outflows in SDSS J1354+1327 is discontinuous AGN outburst activity, which has been seen in simulations and can create outbursts on opposite sides of the galaxy such as those seen in SDSS J1354 +1327 (Hopkins \& Quataert 2010; Gabor \& Bournaud 2014). Clumpy structure in the ISM can lead to highly variable accretion rates onto the SMBH (e.g., Hickox et al. 2014), where each high-accretion event produces an AGN outburst. This outburst injects thermal energy into the ambient gas, launching an outflow. Initially, the heated gas encounters and shocks cold gas, making shocks an indicator of a recent AGN outburst (Gabor \& Bournaud 2014). After this initial period of fast expansion and shocks, the outflow slows and photoionization of the surrounding gas becomes the dominant emission source. Consequently, two sequential AGN outbursts can produce asymmetric outflows; the first AGN outburst leaves behind a large photoionized cone of emission, while the later AGN outburst produces small-scale shock emission.

The observations support this picture of two sequential AGN outbursts. We interpret the southern outflow as the result of an older AGN outburst, based on the photoionized nature of the gas (indicated by the optical line flux ratios and the gas's conical morphology) and the large spatial extent $(\sim 10 \mathrm{kpc})$ of the gas. We interpret the northern outflow as a new outflow that is being driven by a recent AGN outburst, based on the shocked nature of the gas (indicated by the optical line flux ratios, the off-nuclear peak in $[\mathrm{O}$ III] $\lambda 5007$, and the gas's looped morphology) and the small spatial extent $(\sim 1 \mathrm{kpc})$ of the gas.

Therefore, the likeliest explanation for the nature of SDSS $\mathrm{J} 1354+1327$ is that it experienced two sequential AGN outbursts. These episodic AGN outbursts could be the result of a galaxy merger that drives discrete accretion events onto the SMBH that are followed by discrete AGN outbursts (e.g., Gabor \& Bournaud 2013). SDSS J1354+1327 has a companion galaxy located $12.5 \mathrm{kpc}$ to the northeast, and the the tidal debris connecting the two galaxies and the spatial offset between the kinematic center of the galaxy and the center of the stellar light suggest that the two galaxies have already had a close pericenter passage. The interaction with the companion galaxy could have triggered nuclear inflows of gas in SDSS J1354+1327, which produced the two episodes of accretion onto the SMBH that led to the two consecutive AGN outbursts.

There have been many other observations of asymmetric AGN outflows (e.g., Shopbell \& Bland-Hawthorn 1998; Fischer et al. 2011; Greene et al. 2012), but the origins of these asymmetries have not yet been pinpointed. The variety of sizes and velocity profiles observed in AGN outflows could be explained by the lifecycle of AGN flickering (Sun et al. 2017). There also exist AGN outflows with little to no indication of present AGN activity (e.g., Tremonti et al. 2007; Förster Schreiber et al. 2014) and many examples of AGN light echoes (e.g., Lintott et al. 2009; Keel et al. 2015; Runnoe et al. 2016), which may signal SMBHs transitioning from the luminous AGN phase to quiescence.

The galaxy that is the most similar to SDSS J1354+1327 is the $z=0.12$ galaxy SDSS J1356+1026. SDSS J1356+1026 also has velocity-offset emission lines in its SDSS spectrum (although they are double peaked, instead of single peaked as for SDSS J1354+1327), large-scale AGN-driven outflows, and a companion galaxy located $3 \mathrm{kpc}$ away (Liu et al. 2010; Fu et al. 2012; Greene et al. 2012, 2014; Comerford et al. 2015). The major difference is that SDSS J1356+1026 is much more luminous (bolometric luminosity $1.5 \times 10^{46} \mathrm{erg} \mathrm{s}^{-1}$ ) and powerful: SDSS J1356+1026 is an ultra-luminous infrared galaxy hosting a quasar that is powering large-scale $(\sim 10 \mathrm{kpc})$ 
symmetric $\sim 1000 \mathrm{~km} \mathrm{~s}^{-1}$ outflows of ionized gas, and it also hosts a compact $(\sim 0.3 \mathrm{kpc})$ structure of molecular gas (Greene et al. 2012; Sun et al. 2014). If the molecular gas is in an outflow, then the outflows in SDSS J1356+1026 may be driven by episodic AGN activity, where the ionized outflows were launched before the molecular outflow (Sun et al. 2014).

We conclude that SDSS J1354+1327 is the galaxy with the strongest evidence for both a light echo of a previous AGN outflow and a new AGN outflow being launched. This is direct observational evidence for the episodic AGN outbursts that are seen in simulations. Given the upper limits on when the outflows launched, and the amount of time that emission can remain visible, we infer that the timescale between the two AGN outbursts was $\lesssim 10^{5}$ years.

\section{Conclusions}

We have used Chandra, HST, APO/DIS optical longslit spectroscopy, and Keck/OSIRIS near-infrared AO-assisted IFS to determine the nature of the $z=0.06$ galaxy SDSS J1354 +1327 . SDSS J1354+1327 was originally selected from SDSS spectra of active galaxies for its narrow AGN emission lines that are offset in line-of-sight velocity from systemic by $69 \mathrm{~km} \mathrm{~s}^{-1}$. We determined that the velocity offset arises from the peak in emission line gas, which is located $0.40 \mathrm{kpc}$ northeast of the galaxy center.

We conclude that SDSS J1354+1327 is a galaxy whose AGN has recently turned off and then on again through two accretion events that led to two sequential AGN outbursts. The two accretion events arose from clumpy ISM being driven to the center of SDSS J1354+1327 as a result of a tidal interaction with the companion galaxy located $12.5 \mathrm{kpc}$ away. The outflow visible to the south of the galaxy center is remnant emission lingering from a past AGN outburst, because it is photoionized gas with a large spatial extent of the gas $(\sim 10 \mathrm{kpc})$. The emission north of the galaxy center is a new AGN outburst that launched a new outflow, based on the shocked nature of the gas and the smaller spatial extent of the gas $(\sim 1 \mathrm{kpc})$. We estimate that the timescale between the AGN outburst that produces the southern outflow and the AGN outburst that is powering the northern outflow is $\lesssim 10^{5}$ years.

SDSS J1354+1327 is the strongest observational example of a galaxy with an AGN that has turned off and then on again. As such, SDSS J1354+1327 fits into the broader picture of episodic AGN activity that also includes many observations of light echoes of past AGN outbursts. Our attention was initially drawn to SDSS J1354+1327 due to its narrow emission lines that are offset in line-of-sight velocity from systemic. These velocity-offset emission lines are commonly indicators of shocks, and one mechanism that produces shocks is a recent AGN outburst being driven into the ISM. Therefore, future analyses of galaxies with velocity-offset AGN emission lines will certainly uncover young AGN outbursts and some fraction of them may have evidence of past AGN activity as well.

J.M.C. thanks Nadia Zakamska for useful discussions. We also thank the anonymous referee for comments that have improved the clarity of this paper. Support for this work was provided by NASA through Chandra Award Number GO4-15113X issued by the Chandra X-ray Observatory Center, which is operated by the Smithsonian Astrophysical Observatory for and on behalf of NASA under contract NAS8-03060. Support for HST program number GO 13513 was provided by NASA through a grant from the Space Telescope Science Institute, which is operated by the Association of Universities for Research in Astronomy, Inc., under NASA contract NAS 5-26555.

The scientific results reported in this article are based in part on observations made by the Chandra X-ray Observatory, and this research has made use of software provided by the Chandra X-ray Center in the application packages CIAO, ChIPS, and Sherpa. The results reported here are also based on observations made with the NASA/ESA Hubble Space Telescope, obtained at the Space Telescope Science Institute, which is operated by the Association of Universities for Research in Astronomy, Inc., under NASA contract NAS 5-26555. These observations are associated with program number GO 13513.

Some of the observations reported here were obtained at the Apache Point Observatory $3.5 \mathrm{~m}$ telescope, which is owned and operated by the Astrophysical Research Consortium.

Some of the data presented herein were obtained at the W.M. Keck Observatory, which is operated as a scientific partnership among the California Institute of Technology, the University of California and the National Aeronautics and Space Administration. The Observatory was made possible by the generous financial support of the W.M. Keck Foundation. The authors recognize and acknowledge the very significant cultural role and reverence that the summit of Mauna Kea has always had within the indigenous Hawaiian community. We are most fortunate to have the opportunity to conduct observations from this mountain.

Facilities: CXO (ACIS), HST (WFC3), APO (Dual Imaging spectrograph), Keck:I (OSIRIS).

\section{ORCID iDs}

R. Scott Barrows (iD https://orcid.org/0000-0002-6212-7328

Rebecca Nevin (iD https://orcid.org/0000-0003-1056-8401

David Pooley (D) https://orcid.org/0000-0003-4897-7833

Daniel Stern (iD https://orcid.org/0000-0003-2686-9241

Fiona A. Harrison (iD https://orcid.org/0000-0003-2992-8024

\section{References}

Allen, J. T., Schaefer, A. L., Scott, N., et al. 2015, MNRAS, 451, 2780 Antonucci, R. R. J., \& Miller, J. S. 1985, ApJ, 297, 621

Bae, H.-J., \& Woo, J.-H. 2016, ApJ, 828, 97

Baldwin, J. A., Phillips, M. M., \& Terlevich, R. 1981, PASP, 93, 5 Barrows, R. S., Comerford, J. M., Greene, J. E., \& Pooley, D. 2016, ApJ, 829,37

Barrows, R. S., Sandberg Lacy, C. H., Kennefick, J., et al. 2013, ApJ, 769, 95 Barrows, R. S., Stern, D., Madsen, K., et al. 2012, ApJ, 744, 7

Bevington, P. R. 1969, Data Reduction and Error Analysis for the Physical Sciences (New York: McGraw-Hill)

Clark, N. E., Tadhunter, C. N., Morganti, R., et al. 1997, MNRAS, 286, 558 Comerford, J. M., Gerke, B. F., Newman, J. A., et al. 2009, ApJ, 698, 956

Comerford, J. M., Gerke, B. F., Stern, D., et al. 2012, ApJ, 753, 42

Comerford, J. M., \& Greene, J. E. 2014, ApJ, 789, 112

Comerford, J. M., Pooley, D., Barrows, R. S., et al. 2015, ApJ, 806, 219

Comerford, J. M., Pooley, D., Gerke, B. F., \& Madejski, G. M. 2011, ApJL, 737, L19

Comerford, J. M., Schluns, K., Greene, J. E., \& Cool, R. J. 2013, ApJ, 777, 64 Cutri, R. M., Skrutskie, M. F., van Dyk, S., et al. 2003, yCat, 2246, 1

Davies, R. I., Mueller-Sánchez, F., Genzel, R., et al. 2007, ApJ, 671, 1388

DeGraf, C., Dekel, A., Gabor, J., \& Bournaud, F. 2017, MNRAS, 466, 1462

Dickey, J. M., \& Lockman, F. J. 1990, ARA\&A, 28, 215

Dopita, M. A., \& Sutherland, R. S. 1996, ApJS, 102, 161

Fabian, A. C. 2012, ARA\&A, 50, 455

Faucher-Giguère, C.-A., \& Quataert, E. 2012, MNRAS, 425, 605 
Ferland, G. J. 1996, Hazy, A Brief Introduction to Cloudy, Vol. 90, Univ. Kentucky Physics and Astronomy Department Internal Report, http:// www.pa.uky.edu/ gary/cloudy

Ferland, G. J., Porter, R. L., van Hoof, P. A. M., et al. 2013, RMxAA, 49, 137 Fischer, T. C., Crenshaw, D. M., Kraemer, S. B., et al. 2011, ApJ, 727, 71

Förster Schreiber, N. M., Genzel, R., Newman, S. F., et al. 2014, ApJ, 787, 38

Fu, H., Yan, L., Myers, A. D., et al. 2012, ApJ, 745, 67

Gabor, J. M., \& Bournaud, F. 2013, MNRAS, 434, 606

Gabor, J. M., \& Bournaud, F. 2014, MNRAS, 441, 1615

Graham, A. W., \& Driver, S. P. 2005, PASA, 22, 118

Greene, J. E., Pooley, D., Zakamska, N. L., Comerford, J. M., \& Sun, A.-L. 2014, ApJ, 788, 54

Greene, J. E., Zakamska, N. L., \& Smith, P. S. 2012, ApJ, 746, 86

Hickox, R. C., Mullaney, J. R., Alexander, D. M., et al. 2014, ApJ, 782, 9

Hopkins, P. F., \& Quataert, E. 2010, MNRAS, 407, 1529

Iono, D., Yun, M. S., \& Mihos, J. C. 2004, ApJ, 616, 199

Ishibashi, W., \& Courvoisier, T.-L. 2010, A\&A, 512, A58

Kauffmann, G., Heckman, T. M., Tremonti, C., et al. 2003, MNRAS, 346, 1055

Keel, W. C., Maksym, W. P., Bennert, V. N., et al. 2015, AJ, 149, 155

Kennicutt, R. C., \& Evans, N. J. 2012, ARA\&A, 50, 531

Kewley, L. J., Dopita, M. A., Sutherland, R. S., Heisler, C. A., \& Trevena, J. 2001, ApJ, 556, 121

Kewley, L. J., Groves, B., Kauffmann, G., \& Heckman, T. 2006, MNRAS, 372,961

Koyama, K., Maeda, Y., Sonobe, T., et al. 1996, PASJ, 48, 249

Krajnović, D., Cappellari, M., de Zeeuw, P. T., \& Copin, Y. 2006, MNRAS, 366,787

Lagarias, J. C., Reeds, J. A., Wright, M. H., \& Wright, P. E. 1998, SIAM Journal on Optimization, 9, 112

LaMassa, S. M., Cales, S., Moran, E. C., et al. 2015, ApJ, 800, 144

Lintott, C. J., Schawinski, K., Keel, W., et al. 2009, MNRAS, 399, 129

Liu, X., Shen, Y., Strauss, M. A., \& Greene, J. E. 2010, ApJ, 708, 427

Liu, X., Shen, Y., Strauss, M. A., \& Hao, L. 2011, ApJ, 737, 101

Malkan, M. A., Gorjian, V., \& Tam, R. 1998, ApJS, 117, 25

Martini, P., Regan, M. W., Mulchaey, J. S., \& Pogge, R. W. 2003, ApJS, 146,353

McGurk, R. C., Max, C. E., Medling, A. M., Shields, G. A., \& Comerford, J. M. 2015, ApJ, 811, 14

Moy, E., \& Rocca-Volmerange, B. 2002, A\&A, 383, 46

Mulchaey, J. S., Wilson, A. S., \& Tsvetanov, Z. 1996, ApJS, 102, 309
Müller Sánchez, F., Davies, R. I., Genzel, R., et al. 2009, ApJ, 691, 749

Müller-Sánchez, F., Comerford, J., Stern, D., \& Harrison, F. A. 2016, ApJ, 830,50

Müller-Sánchez, F., Comerford, J. M., Nevin, R., et al. 2015, ApJ, 813, 103

Nandra, K., \& Pounds, K. A. 1994, MNRAS, 268, 405

Nevin, R., Comerford, J., Müller-Sánchez, F., Barrows, R., \& Cooper, M. 2016, ApJ, 832, 67

Nevin, R., Comerford, J. M., Müller Sánchez, F., Barrows, R. S., \& Cooper, M. C. 2017, MNRAS, in press (arXiv:1710.00828)

Norman, C. A., \& Ikeuchi, S. 1989, ApJ, 345, 372

Oh, K., Sarzi, M., Schawinski, K., \& Yi, S. K. 2011, ApJS, 195, 13

Oh, K., Yi, S. K., Schawinski, K., et al. 2015, ApJS, 219, 1

Osterbrock, D. E., \& Ferland, G. J. 2006, Astrophysics of Gaseous Nebulae and Active Galactic Nuclei (Mill Valley, CA: Univ. Science Books)

Park, T., Kashyap, V. L., Siemiginowska, A., et al. 2006, ApJ, 652, 610

Peng, C. Y., Ho, L. C., Impey, C. D., \& Rix, H.-W. 2010, AJ, 139, 2097

Piconcelli, E., Jimenez-Bailón, E., Guainazzi, M., et al. 2005, A\&A, 432, 15

Reeves, J. N., \& Turner, M. J. L. 2000, MNRAS, 316, 234

Richardson, C. T., Allen, J. T., Baldwin, J. A., Hewett, P. C., \& Ferland, G. J. 2014, MNRAS, 437, 2376

Runnoe, J. C., Cales, S., Ruan, J. J., et al. 2016, MNRAS, 455, 1691

Schawinski, K., Koss, M., Berney, S., \& Sartori, L. F. 2015, MNRAS, 451, 2517

Schirmer, M., Malhotra, S., Levenson, N. A., et al. 2016, MNRAS, 463, 1554

Schmitt, H. R., Donley, J. L., Antonucci, R. R. J., Hutchings, J. B., \& Kinney, A. L. 2003, ApJS, 148, 327

Schnorr-Müller, A., Storchi-Bergmann, T., Robinson, A., Lena, D., \& Nagar, N. M. 2016, MNRAS, 457, 972

Shopbell, P. L., \& Bland-Hawthorn, J. 1998, ApJ, 493, 129

Stern, D., Assef, R. J., Benford, D. J., et al. 2012, ApJ, 753, 30

Su, M., Slatyer, T. R., \& Finkbeiner, D. P. 2010, ApJ, 724, 1044

Sun, A.-L., Greene, J. E., \& Zakamska, N. L. 2017, ApJ, 835, 222

Sun, A.-L., Greene, J. E., Zakamska, N. L., \& Nesvadba, N. P. H. 2014, ApJ, 790, 160

Sutherland, R. S., Bicknell, G. V., \& Dopita, M. A. 1993, ApJ, 414, 510

Tremonti, C. A., Moustakas, J., \& Diamond-Stanic, A. M. 2007, ApJL, 663, L77

Veilleux, S., Cecil, G., \& Bland-Hawthorn, J. 2005, ARA\&A, 43, 769

Veilleux, S., \& Osterbrock, D. E. 1987, ApJS, 63, 295

Wilson, A. S., \& Tsvetanov, Z. I. 1994, AJ, 107, 1227

Zhang, S., Hailey, C. J., Mori, K., et al. 2015, ApJ, 815, 132 